\title{
Frequency and Antimicrobial Susceptibility of Diarrheagenic Escherichia coli Obtained from Patients with Acute Diarrhea in a Tertiary Care Hospital, Bangladesh.
}

\author{
*Kabir MR ${ }^{1}$, Hossain MA ${ }^{2}$, Alam MM³ ${ }^{3}$ Paul SK ${ }^{4}$, Begum $Z^{5}$, Parvin US $^{6}$, \\ Haque $\mathbf{N}^{7}$, Kobayashi $\mathbf{N}^{8}$
}

\begin{abstract}
Escherichia coli (E. coli) is an important group of pathogens associated with diarrhea among children. Despite the fact that diarrhaegenic Escherichia coli (DEC) has been identified as a major etiologic agent of childhood diarrhea, only a few studies have been performed in Bangladesh to identify these organisms. The objective of this study was to determine the frequency and antimicrobial susceptibility of DEC obtained from patients with acute diarrhea. To detect DEC in patients with acute diarrhea, a total of 300 stool specimens were tested by multiplex polymerase chain reaction (PCR). The antimicrobial susceptibility of DEC were tested by Kirby-Bauer disc diffusion technique as per recommendation of CLSI (Clinical and Laboratory Standards Institute), 2010. Out of 300 stool specimens collected from patients with acute diarrhea, the DEC was detected in 18\% (54/300) cases. The dominating strain was Enterotoxigenic E. coli ( ETEC) $(13 \%, 39 / 300)$, followed by Enteroaggregative E. coli (EAEC) (5\%, 15/300) and no Enterohemorrhagic E. coli (EHEC), Enteroinvasive E. coli ( EIEC) and Enteropathogenic E. coli ( EPEC) could be detected. Detected ETEC were $100 \%$ sensitive to Ceftriaxone, Nitrofurantioin, Amikacin, 94\% sensitive to Nalidixic acid, $89 \%$ sensitive to Gentamycin, $83 \%$ sensitive to Ciprofloxacin, $79 \%$ sensitive to Cephalexin, 39\% sensitive to Amoxycillin, $46 \%$ sensitive to Tetracyclin and $31 \%$ sensitive to Cotrimoxazole. Detected EAEC were $100 \%$ sensitive to Ceftriaxone, Nitrofurantioin, Amikacin, Nalidixic acid, 90\% sensitive to Gentamycin and Ciprofloxacin, $85 \%$ sensitive to Cephalexin, 41\% sensitive to Amoxycillin, $49 \%$ sensitive to Tetracycline and $31 \%$ sensitive to Cotrimoxazole. Both ETEC and EAEC isolates exhibited decreased susceptibility for Amoxycillin, Tetracycline and Cotrimoxazole. Our results revealed that ETEC and EAEC, had significant association with acute diarrhea and should be considered as potential pathogens. Guidelines for appropriate use of antibiotics in tertiary care hospitals need updating.
\end{abstract}

C CBMJ 2013 July: Vol. 02 No. 02 P: 46-51

Key words: Acute diarrhea, Diarrhaegenic Escherichia coli, Multiplex PCR, Antimicrobial Susceptibility.

1. $\quad{ }^{*}$ Dr. Md. Rashedul Kabir

Assistant Professor of Microbiology,

Comunity Based Medical College, Bangladesh.

2. Prof. Md. Akram Hossain

Professor \& Head, Department of Microbiology,

Mymensingh Medical College.

3. Prof. Dr. Md. Murshed Alam

Professor (C.C) \& Head, Department of

Microbiology, Comunity Based Medical College, Bangladesh.

4. Dr. Shyamal Kumar Paul

Associate Professor, Department of Microbiology, Mymensingh Medical College.

5. Dr. Zohra Begum

Associate Professor, Department of Microbiology, Comunity Based Medical College, Bangladesh.
6. Dr. Ummey Shanaz Parvin

Assistant Professor, Department of Microbiology, East West Medical College, Dhaka.

7. Dr. Nazia Haque Medical Officer, Department of Microbiology, Mymensingh Medical College.

8. Nobumichi Kobayashi

Professor, Sapporo Medical University, Japan

* Address of correspondence

Mobile: +088-01711327013

E-mail: dr.pipul@yahoo.com 


\section{Introduction}

Acute diarrhea is a major cause of morbidity and mortality worldwide. The acute diarrhea remains a major public health challenge, especially in developing countries where it is a leading cause of death. Every year nearly 1.4 billion episodes of acute diarrhea occur in children of less than 5 years of age in developing countries ${ }^{1}$. It has been estimated that the mean number of episodes of diarrhea per year in children of under 5 years of age from a developing region is $3.2^{1}$. In addition diarrheal illness account for an estimated 12600 deaths each day in children of under 5 years of age in Asia, Africa and Latin America $^{2}$. Kosek and associates (2003) reviewed studies from 1990 to 2000 and concluded that diarrhea accounts for $21 \%$ of all deaths at under five years of age causing 2.5 million death per year in developing countries ${ }^{1}$.

A diversity of recognized microorganisms such as bacteria, viruses and parasites can be associated with severe acute diarrhea in children $^{3}$. Numerous studies performed in different countries have reported diarrheagenic $E$. coli (DEC) as being the most frequent and important among bacterial pathogens associated with acute diarrhea in developing countries. However, the frequencies of these pathogens vary with geographic region and depend on the socioeconomic/sanitary conditions ${ }^{4}$.

In Bangladesh, the acute diarrhea remains one of the most important health problems. One third of the total child death burden is due to diarrhea. Every year, a rural child suffers on average from 4.6 episodes of diarrhea, from which about 230,000 children $\mathrm{die}^{5}$. The DEC has been reported to be responsible for $34 \%$ of diarrheal diseases in Bangladesh $^{6}$.

There are now at least six types of diarrheagenic strains of $E$. coli on the basis of distinct epidemiology and clinical feature, special virulence determinants and association with certain serotypes. There are
Enterotoxigenic
E. coli (ETEC),

Enteropathogenic

E. coli (EPEC),
Enterohemorrhagic E. coli (EHEC),

Enteroaggregative E. coli (EAEC),

Enteroinvasive E. coli (EIEC) and Diffusely adhering $E$. coli (DAEC). Of these, EPEC, ETEC, EIEC, EHEC and EAEC are clearly associated with different types of enteritis, while the DAEC is potential pathogens but its association with diarrhea has not been clearly assessed and further studies are required to confirm its etiological role in diarrheal diseases ${ }^{3,7}$.

Due to lack of facilities, the DEC cannot be detected in the routine diagnostic microbiology laboratory in developing countries, which is important in understanding the disease spectrum, tracing the sources of infection and the burden of the disease. Such identification would also assist the clinician to dispense appropriate management ${ }^{8}$.

Therefore, we carried out this study to determine the frequency and antimicrobial susceptibility of DEC obtained from patients with acute diarrhea.

\section{Methods}

This cross-sectional study was carried out during the period from July' 2011 to December' 2011 in the department of Microbiology, Mymensingh Medical College and included all patients with acute diarrhea irrespective of age and sex, admitted in Mymensingh Medical College Hospital. A total of 300 stool specimens were examined by standard laboratory methods for identification of $E$. coli. Different DEC strains were detected by Multiplex PCR following standard methods. DNA of $E$. coli was extracted from few freshly isolated colonies grown on MAC plates mixed in $100 \mu \mathrm{l}$ of sterile deionized water, by boiling at $100^{\circ} \mathrm{C}$ for 10 minutes and centrifuged supernatant was used as DNA template ${ }^{8}$.

Multiplex PCR for categorization of $E$. coli into EAEC, ETEC, EPEC, STEC and EIEC was done using primers for identification of aggR, CVD432 and aspU genes for EAEC, elt or est gene for ETEC, eae gene for EPEC, eae or stx genes for STEC and ipaH gene for EIEC (Table: 1$)^{8,9}$. The PCR amplification was carried out with a $50 \mu \mathrm{l}$ reaction mixture 
[buffer, dNTP, Primers, Taq DNA polymerase, nuclease free water and DNA tamplate] using the following thermal and cycling conditions: initial denaturation at $95^{\circ} \mathrm{C}$ for $5 \mathrm{~min}$, followed by 30 cycles, each containing denaturation at $95^{\circ} \mathrm{C}$ for $1 \mathrm{~min}$, annealing at $56^{\circ} \mathrm{C}$ for $1 \mathrm{~min}$, and extension at $72^{\circ} \mathrm{C}$ for $1 \mathrm{~min}$; and final extension at $72^{\circ} \mathrm{C}$ for $10 \mathrm{~min}$. The amplified products were then separated by horizontal electrophoresis on a 1.0\% agarose gel, stained with ethidium bromide and visualized under UV Trans- illuminator ${ }^{8,9}$.

Antimicrobial susceptibility testing by disc diffusion method:

All the isolates were put into antibiotic susceptibility test by Kirby-Bauer disc diffusion technique as per recommendation of CLSI (Clinical and Laboratory Standards Institute), 2010. Panel of antibiotics were used. All tests were performed on MullerHinton agar. The surface was lightly and uniformly inoculated by cotton swab. Prior to inoculation, the swab stick was dipped into bacterial suspension having visually equivalent turbidity to 0.5 McFarland standards. The swab stick was then took out and squeezed on the wall of the test tube to discard extra suspension. Inoculated plates were incubated at $37{ }^{\circ} \mathrm{C}$ for 24 hours. On the next day, plates were read by taking measurement of zone of inhibition. Results were recorded and graded as Resistant (R) and Sensitive (S) according to the reference zone of inhibition of particular antibiotic (CLSI, 2010). Known control strain ATCC, No. 25922 and ATCC No. 25923 were used for quality control $^{10,11}$.

\section{Antimicrobial agents used}

A total of 10 antimicrobial agents were used for determining antibiogram of isolated organisms according to Gram negative panel recommended by CLSI, 2010. Antibiotics were: Amoxycillin, Cephalexin, Ciprofloxacin, Gentamicin, Amikacin, Nalidixic acid, Ceftriazone, Cotrimoxazole, Nitrifurantioin and Tetracyclin ${ }^{10,11}$.
Table I: PCR primers were used for detecting different diarrheagenic Escherichia coli in the present study.

\begin{tabular}{|c|c|c|}
\hline Designation-Sequence(5 to 3)- & \multicolumn{2}{|c|}{ Target gene- Amplicon size(bp) } \\
\hline $\begin{array}{l}\text { SK1 CCC GAA TTC GGC ACA AGC ATA AGC } \\
\text { SK2 CCC GGA TCC GTC TCG CCA GTA TTC G }\end{array}$ & eae & 881 \\
\hline $\begin{array}{l}\text { VTcom-u GAG CGA AAT AAT TTA TAT GTG } \\
\text { VTcom-d TGA TGA TGG CAA TTC AGT AT }\end{array}$ & stx & 518 \\
\hline $\begin{array}{l}\text { AL } 65 \text { TTA ATA GCA CCC GGT ACA AGC AGG } \\
\text { AL125 CCT GAC TCT TCA AAA GAG AAA ATT AC }\end{array}$ & est & 147 \\
\hline $\begin{array}{l}\text { LTL TCT CTA TGT GCA TAC GGA GC } \\
\text { LTR CCA TAC TGA TTG CCG CAA T }\end{array}$ & elt & 322 \\
\hline $\begin{array}{l}\text { ipalll GTT CCT TGA CCG CCT TTC CGA TAC CGTC } \\
\text { ipalV GCC GGT CAG CCA CCC TCT GAG AGT AC }\end{array}$ & ipaH & 619 \\
\hline $\begin{array}{l}\text { aggRks1 GTA TAC ACA AAA GAA GGA AGC } \\
\text { aggRkas2 ACA GAA TCG TCA GCA TCA GC }\end{array}$ & $\operatorname{aggR}$ & 254 \\
\hline $\begin{array}{l}\text { Eaggfp AGA CTC TGG CGA AAG ACT GTA TC } \\
\text { Eaggbp ATG GCT GTC TGT AAT AGA TGA GAAC }\end{array}$ & CVD432 & 194 \\
\hline aspU-3 GCC TTT GCG GGT GGT AGC GG & aspU & 282 \\
\hline
\end{tabular}

aspU-2 AAC CCA TTC GGT TAG AGC AC

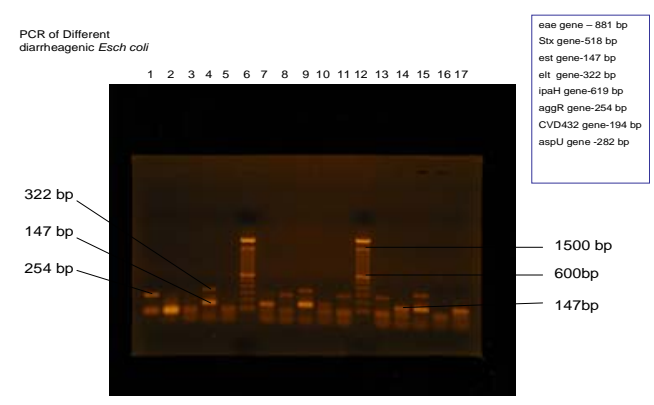

Photograph of multiplex PCR showing aggR gene (254 bp) of EAEC in lane 1, est gene (147 bp) and elt gene (322 bp) of ETEC in lane 4, only est gene (147 bp) of ETEC in lane 14 and ladder marker (100bp) in lane 5 and 12.

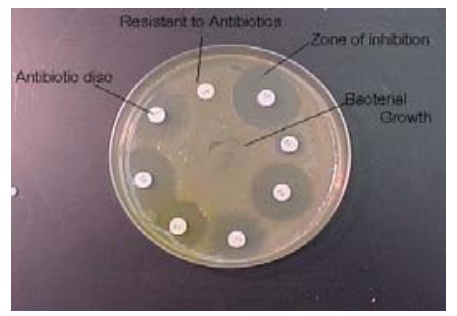


Photograph of antibiotic susceptibility test of isolated ETEC by Kirby-Bauer disc diffusion technique on Muller-Hinton agar medium.

\section{Results}

In the present study, majority of the cases $(62 \%)$ belonged to $<5$ years of age, in which $18 \%(54 / 300)$ cases were in the age group $<1$ year and $44 \%(132 / 300)$ cases were in the age group 1-5 years. The rest 38\% (114/300) cases were in the age group $>5$ years (Figure: I).

Of the 300 specimens examined, the DEC was detected in $18 \%(54 / 300)$ cases. The dominating strain was ETEC (13\%, 39/300), followed by $\operatorname{EAEC~}(5 \%, 15 / 300)$ and no EHEC, EIEC and EPEC could be detected (Table: II).

Detected ETEC were $100 \%$ sensitive to Ceftriaxone, Nitrofurantioin, Amikacin, 94\% sensitive to Nalidixic acid, $89 \%$ sensitive to Gentamycin, 83\% sensitive to Ciprofloxacin, $79 \%$ sensitive to Cephalexin, 39\% sensitive to Amoxycillin, $46 \%$ sensitive to Tetracyclin and $31 \%$ sensitive to Cotrimoxazole(Table:III). Detected EAEC were $100 \%$ sensitive to Ceftriaxone, Nitrofurantioin, Amikacin, Nalidixic acid, 90\% sensitive to Gentamycin and Ciprofloxacin, $85 \%$ sensitive to Cephalexin, $41 \%$ sensitive to Amoxycillin, $49 \%$ sensitive to Tetracycline and $31 \%$ sensitive to Cotrimoxazole(Table: IV). Both ETEC and EAEC isolates exhibited decreased susceptibility for Amoxycillin, Tetracycline and Cotrimoxazole.

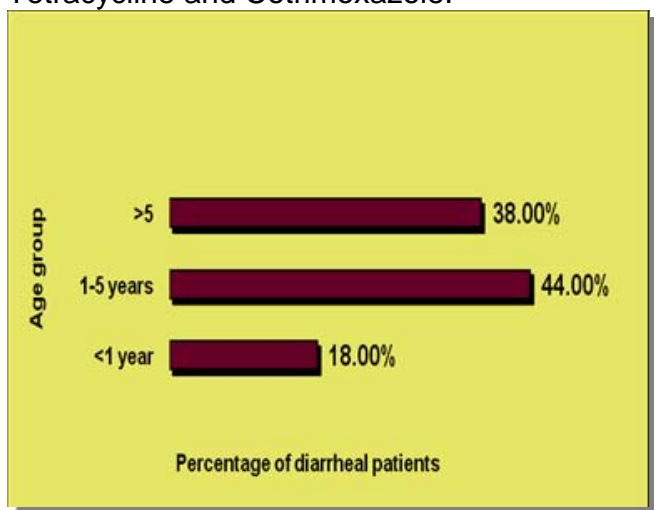

Figure I: Age distribution of the study population.
Table II: Distribution of different diarrheagenic Escherichia coli (DEC) Strains in the study population $(n=300)$.

\begin{tabular}{|c|c|}
\hline $\begin{array}{c}\text { Diarrheagenic } \\
\text { Escherichia coli }\end{array}$ & $\begin{array}{c}\text { Number of } \\
\text { cases (\%). }\end{array}$ \\
\hline ETEC & $39(13)$ \\
\hline EAEC & $15(5)$ \\
\hline EPEC & $0(0)$ \\
\hline EIEC & $0(0)$ \\
\hline EHEC & $0(0)$ \\
\hline Total & $54(18)$ \\
\hline
\end{tabular}

Table III: Antibiotic sensitivity patterns of Enterotoxogenic E. coli (ETEC) isolated from the stool samples $(n=39)$.

\begin{tabular}{|l|c|}
\hline Antibiotic & $\begin{array}{c}\text { Number of ETEC } \\
\text { strains sensitivity to } \\
\text { different antibiotics (\%). }\end{array}$ \\
\hline Ceftriaxone & $39(100)$ \\
\hline Amikacin & $39(100)$ \\
\hline Nitrofurantioin & $39(100)$ \\
\hline Nalidixic acid & $36(94)$ \\
\hline Gentamycin & $34(89)$ \\
\hline Ciprofloxacin & $32(83)$ \\
\hline Cephalexin & $30(79)$ \\
\hline Tetracycline & $18(46)$ \\
\hline Amoxycillin & $15(39)$ \\
\hline Cotrimoxazole & $12(31)$ \\
\hline
\end{tabular}

Table IV: Antibiotic sensitivity patterns of Enteroaggregative $E$. coli (EAEC) isolated from the stool samples $(n=15)$.

\begin{tabular}{|l|c|}
\hline Antibiotic & $\begin{array}{c}\text { Number of EAEC } \\
\text { strains sensitivity to } \\
\text { different antibiotics (\%). }\end{array}$ \\
\hline Ceftriaxone & $15(100)$ \\
\hline Amikacin & $15(100)$ \\
\hline Nalidixic acid & $15(100)$ \\
\hline Nitrofurantioin & $15(100)$ \\
\hline Ciprofloxacin & $13(90)$ \\
\hline Gentamycin & $13(90)$ \\
\hline Cephalexin & $12(85)$ \\
\hline Tetracyclin & $7(49)$ \\
\hline Amoxycillin & $6(41)$ \\
\hline Cotrimoxazole & $5(31)$ \\
\hline
\end{tabular}




\section{Discussion}

Acute diarrhea is one of the most common illness and cause of death in young children in Bangladesh. In the present study, majority of the cases (62\%) belonged to $<5$ years of age, in which $18 \%$ cases were in the age group $<1$ year and $44 \%$ cases were in the age group 1-5 years. The rest $38 \%$ cases were in the age group $>5$ years. Albert and associates (1999) as well as Stoll and associates (1982) reported that diarrhea was more common in children of $<5$ years of age in Bangladesh ${ }^{12,13}$, which supports the present study findings. In a recent study from Vietnam by Nguyen and associates (2005), it was found that diarrhea was more frequent in children of less than 5 years of age $^{2}$, which correlates with the present study findings.

In the present study, the DEC was detected in 18\% (54/300) cases (Table II). In 2002 and 1999 , it was reported that DEC was responsible for $34 \%$ and $26 \%$ of diarrheal diseases in Bangladesh respectively ${ }^{6,12}$. All the above study findings were higher than the present study. Various factors might be responsible for such a difference In the present study, the samples collected were neither directly inoculated in culture media nor transported by transport media, history of taking antibiotics before sample collection and seasonality might be important factors to reduce the identification of DEC in the study population $^{13}$. In Vietnum, Nguyen and associates (2005) and in Mozambique, Rappelli and associated (2005) found DEC was responsible for $22.5 \%$ and $20 \%$ of diarrheal diseases respectively ${ }^{2,7}$, which correlate well with the present study findings. In the present study, among the diarrheagenic $E$. coli (DEC), the ETEC was detected in $72 \%(39 / 54)$ cases and the EAEC was detected in $28 \%(13 / 54)$ cases. No EHEC, EPEC and EIEC could be detected. The ETEC was found to be the most prevalent DEC in the study population. In 2002, it was reported that ETEC was most prevalent DEC, responsible for diarrheal diseases in Bangladesh which supports the present study results ${ }^{14}$. The EAEC was the second most prevalent DEC in the present study population. EAEC has been found as a common diarrheal pathogen in children in many developing countries ${ }^{5}$. The EPEC could not be detected in the present study population. The most important feature of the diarrheal diseases due to EPEC infection is the remarkable age distribution. The EPEC infection is primarily a disease of infants younger than 1 year of age $\mathrm{e}^{16}$. In the present study, only $18 \%$ cases were found in the age group below 1 year. Nessa and associates (2007) as well as Unicomb and associates (1996) could not detect EPEC from any age group in Bangladesh ${ }^{8,17}$. In the present study, the EIEC and EHEC could not be detected in the study population. In many previous studies carried out in Bangladesh, the EIEC and EHEC could not be detected in diarrheal patients $^{18,19,20,21}$. The absence of these strains is not surprising since these pathotypes are not frequently detected in developing countries of Africa and Asia ${ }^{7}$.

In the present study, detected ETEC were $100 \%$ sensitive to Ceftriaxone, Nitrofurantioin, Amikacin, 94\% sensitive to Nalidixic acid, $89 \%$ sensitive to Gentamycin, $83 \%$ sensitive to Ciprofloxacin, $79 \%$ sensitive to Cephalexin, $39 \%$ sensitive to Amoxycillin, $46 \%$ sensitive to Tetracyclin and $31 \%$ sensitive to Cotrimoxazole (Table: III). Detected EAEC were $100 \%$ sensitive to Ceftriaxone, Nitrofurantioin, Amikacin, Nalidixic acid, 90\% sensitive to Gentamycin and Ciprofloxacin, $85 \%$ sensitive to Cephalexin, $41 \%$ sensitive to Amoxycillin, $49 \%$ sensitive to Tetracycline and $31 \%$ sensitive to Cotrimoxazole (Table: IV). Both ETEC and EAEC isolates exhibited decreased susceptibility for Amoxycillin, Tetracycline and Cotrimoxazole. This trend agrees with the findings in other study in Germany $^{22}$. kalantar and associates (2011) found similar type of results that supports the present study findings ${ }^{23}$.

\section{Conclusion}

We therefore, recommend the routine isolation and identification of E.coli strains form the patients with acute diarrhea and application of appropriate use of antibiotics and updating guidelines for appropriate use of antibiotics in tertiary care hospitals . 
Original Article

\section{Acknowledgement}

We are grateful to Professor Nobumichi Kobayashi, Sapporo Medical University of Japan for providing with us primers of PCR and technical assistance for this study.

\section{References:}

1. Kosek M, Bern C and Guerrant RL. The global burden of diarrhoeal disease, as estimated from studies published between 1992 and 2000. Bull World Health Organ. 2003; 81: 197-204.

2. Nguyen RN, Taylor LS and Tauschek $M$. Atypical enteropathogenic Escherichia coli infection and prolonged diarrhea in children. Emerg Infect Dis. 2006; 12(4): 597-603.

3. Al-Gallas $N$, Abbassi SM, Hassan $A B$ and Aissa RB. Genotypic and phenotypic profiles of enterotoxigenic Escherichia coli associated with acute diarrhea in Tunis, Tunisia. Curr Microbiol. 2007; 55: 47-55.

4. O'Ryan M, Lucero $Y$, O'Ryan-Sorian, MA and Ashkenazi S. An update on management of severe acute infectious gastroenteritis in children. Expert Rev Anti Infect. 2011; 8: 671682.

5. Piechulek $H$, Al-Sabbir $A$ and MendozaAldana J. Diarrhea and ARI in rural areas of Bangladesh. J Trop Med. 2003; 34(2): 337342.

6. ICDDR, B. Trends in Aetiologies for Diarrhoeal Diseases. Health Scien Bull 2002; 1(1): 12 15.

7. Rappelli $P$, Folgosa $E$, Solinas ML, DaCosta $J L$ and Colombo MM. Pathogenic enteric Escherichia coli in children with and without diarrhea in Maputo, Mozambique. FEMS Immun Med Microbiol. 2005; 43: 67-72.

8. Nessa k, Dilruba A, Islam $J$, Kabir $L$ and Hossai, MA. Usefulness of a Multiplex PCR for Detection of Diarrheagenic Escherichia coli in a Diagnostic Microbiology Laboratory Setting, Bangladesh. J Med Microbiol. 2007; 1(2): 38-42.

9. Toma C, Lu Y, Higa N, Nakasone N, Rivas $M$ and Iwanaga M. Multiplex PCR Assay for Identification of Human Diarrheagenic Escherichia coli. J Clin Microbiol. 2003; 41(6): 2669-2671.

10. Bauer AW, Kirby WM, Sherris JC and Turck M. Antibiotic susceptibility testing by a standardized single disk method. Am J. Clin. Path. 1996; 45(2): 493 - 501.

11. Clinical and Laboratory Standards Institute. 'Performance standards for antimicrobial susceptibility testing'. Seventeenth Informational Supplement.2010: M 100-S17.
12. Albert MJ, Faruque $A$, Faruque $G$, Sack $B$ and Mahalian D. Case-Control Study of Enteropathogens Associated with Childhood Diarrhea in Dhaka, Bangladesh. J Clin Microbiol. 1999; 37(11): 3458-3464.

13. Stoll BJ. The global impact of neonatal infection. Clin Perinatol. 1997; 24: 1-21.

14. Qadri F, Das SK, Faruque G, Fuchs J, Albert MJ and Svennerholm AM. Prevalence of Toxin Types and Colonization Factors in Enterotoxigenic Escherichia coli Isolated during a 2-Year Period from Diarrheal Patients in Bangladesh. J Med Microbiol. 2000; 38(1): 27-31.

15. Faruque SM, Haider K, Albert MJ, Ahmad S and Alam N. A comparative study of specific gene probes and bioassays to identify diarrhoeagenic Escherichia coli in paediatric patients with diarrhoea in Bangladesh. J Med Microbiol. 1992; 36: 37-40.

16. Nataro JP \& Karper JB. Diarrheagenic Escherichia coli. Clin Microbial Rev. 1998; 11:142-201.

17. Unicomb LE, Faruque SM, Malek MA, Faruque $A G$ and Albert MJ. Demonstration of a Lack of Synergistic Effect of Rotavirus with Other Diarrheal Pathogens on Severity of Diarrhea in Children. J Clin Microbiol. 1996; 34(5): 13401342.

18. Baqui $A H$, Sack RB, Black RE, Haider $K$, Hossain A, Yunus M, Chowdhury HR and Siddique AK. Enteropathogens Associated with Acute and Persistent Diarrhea in Bangladeshi Children <5 Years of Age. J Infec Dis. 1992; 166: 792-796.

19. Albert MJ, Faruque SM, Ansaruzzaman $M$, Bhuiyan NA, Alam K and Akbar MS. Controlled Study of Escherichia coli Diarrheal Infections in Bangladeshi Children, J Clin Microbiol. 1995; 33(4): 973-977.

20. Strockbine NA, Faruque SM, Alam K, Tzipori S and Wachsmuth. DNA probe analysis of diarrhoeagenic Escherichia coli: detection of EAF-positive isolates of traditional enteropathogenic E. coli serotypes among Bangladeshi paediatric diarrhea patients. Mol Cell Probes. 1992; 6: 93-99.

21. Qadri F, Ashraful IK, Syed A, Faruque G, Begum YA, Chowdhury $F$ and Svennerholm AM. Enterotoxigenic Escherichia coli and Vibrio cholerae Diarrhea, Bangladesh, 2004. Emerg infect dis. 2005; 11(7)1104-1006.

22. Presterl E, Zwick RK, Reichiman $S$ and Alcheburg A.Frequency and virulence properties of diarrheagenic Escherchia coli in children with diarrhea in Gabon. J. Trop. Med. Hyg.2003: 69 (4):406-410.

23. Kalantar $E$, Soheili $F$, Salimi $H$, Soltan $D$ and allal MM. Antimicrobial susceptibility and plasmid profiles of Escherichia coli pathotypes obtained from children with acute diarrhea. Jundishapur J Microbial.2011; 1(4):23-28. 